\title{
Belphégor
}

\section{Le Policier fantôme. Entretien de Jan Baetens avec Luc Dellisse le 4 novembre 2020}

Jan Baetens

\section{(2) OpenEdition}

12 Journals

\section{Electronic version}

URL: https://journals.openedition.org/belphegor/4040

DOI: 10.4000/belphegor.4040

ISSN: 1499-7185

Publisher

LPCM

\section{Electronic reference}

Jan Baetens, "Le Policier fantôme. Entretien de Jan Baetens avec Luc Dellisse le 4 novembre 2020", Belphégor [Online], 19-1 | 2021, Online since 24 June 2021, connection on 08 July 2021. URL: http:// journals.openedition.org/belphegor/4040 ; DOI: https://doi.org/10.4000/belphegor.4040

This text was automatically generated on 8 July 2021.

\section{(c) (i) (9)}

Belphégor est mis à disposition selon les termes de la Licence Creative Commons Attribution - Pas d'Utilisation Commerciale - Pas de Modification 4.0 International. 


\section{Le Policier fantôme. Entretien de Jan Baetens avec Luc Dellisse le 4 novembre 2020}

Jan Baetens

\section{REFERENCES}

Luc Delisse. Le Policier fantôme. Bruxelles. Espace Nord. 2017. 400 p. Edition entièrement revue et augmentée. ISBN: 978-2-87568-139-3 


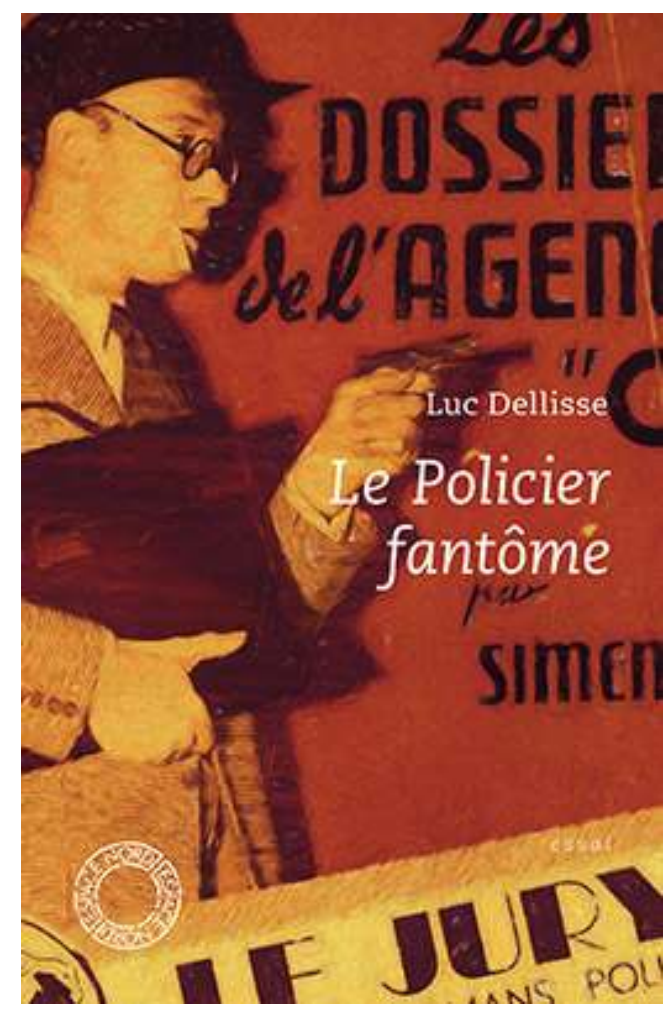

1 Jan Baetens : Le Policier fantôme est l'étude très détaillée d'un type particulier de roman policier, le récit de détection tel qu'il fut produit, c'est-à-dire écrit, publié et lu à une grande échelle, durant la Deuxième Guerre mondiale.

2 Republié trente-trois ans après sa première parution en 1984, le livre se voit ici radicalement élargi et nuancé, car vous y avez inclus non seulement la production plus récente voire contemporaine, mais aussi certaines productions en langue néerlandaise. L'accent mis sur la spécificité de ce que vous décrivez comme le récit policier belge " traditionnel» vous a obligé à faire un choix terminologique radical : vous maintenez le terme de " récit policier ", au détriment du terme de "polar ", que vous récusez pour le corpus en question.

3 Luc Dellisse : Le mot polar, qui désigne aujourd'hui indifféremment tout type de roman policier, mérite d'être interrogé. Il me semble que c'est un tort de l'appliquer à n'importe quelle fiction policière, des origines jusqu'à aujourd'hui. En réalité il est extrêmement connoté, par rapport à la fois à une époque et à une catégorie particulière du roman noir.

4 Il en va un peu du mot polar comme du mot bouquiner. On peut essayer de l'utiliser comme simple synonyme de lire, mais cet usage omnivalent du mot ne fonctionne pas vraiment. Dire « je bouquine un peu le soir » a un sens s'il s'agit d'un roman de série ou d'un récit de vie, mais devient étrange, voire absurde, appliqué à l'œuvre de Joyce ou de Kant. De la même façon, dire " je me suis mis au lit avec un bon polar », pour qualifier un récit d'énigme classique, disons Le Mystère de la chambre jaune ou un roman d'Agatha Christie, révèle les limites du terme.

5 C'étaient ces réflexions toutes simples que je me faisais, au moment de m'intéresser de plus près à la fortune singulière du roman policier belge durant la Seconde Guerre mondiale, dans les circonstances imprévues de l'Occupation. Le terme polar m'aurait semblé comique, inapproprié, et d'une certaine manière, totalement anachronique, si je m'en étais servi pour évoquer des ouvrages de détection classique, caractérisés par un 
système lent, profond, rationnel, prospectif, codé et résolument non politique, qui était la norme du récit d'aventure, d'enquête ou de mystère en faveur à ce moment-là.

6 Jan Baetens : Polar et récit policier sont donc pour vous deux formes d'écriture, voire deux genres presque différents?

7 Luc Dellisse : Il me semble qu'intrinsèquement, le mot polar est lié aux circonstances sociologiques et culturelles de son apparition. C'est un terme qui doit surgir au tout début des années 70 . Il a comme auteur de référence, presque comme auteurprescripteur, celui dont on peut dire que non seulement il a écrit spécifiquement des polars mais qu'il en est l'inventeur. Non du terme mais du genre: Jean-Patrick Manchette. Cet écrivain fulgurant a renouvelé la vision du roman policier de langue française, autant que deux générations avant lui, Chandler avait pu le faire pour le roman policier américain: en y apportant un mélange de décalage, d'absence, de dureté, de conduite d'échec, de description pessimiste de la société, de prise en compte de la réalité politique, morale et en somme mafieuse de notre univers; et en même temps, en faisant entendre, comme un chant de sirène, une sorte de rythme alterné de gravité et de douceur qui est le propre d'un styliste conscient de son art. Cet univers-là s'est tout de suite confondu avec l'idée générique de polar, et lui a donné son succès, sa nécessité et sa vérité. Mais en filigrane il y a toute une époque - celle de Giscard d'Estaing pour l'essentiel - et elle a coulé à pic, laissant le vocable polar seul devant son miroir.

8 Il paraît dès lors utile de disposer d'au moins deux termes spécifiques: "roman policier » et «polar » pour qualifier des genres et dans une large mesure des époques distincts. Ce n'est d'ailleurs pas uniquement une question de paradigme. Il n'est pas certain que désormais, un ouvrage de fiction policière doive forcément être qualifié de polar, sous prétexte qu'il est paru après 1970. Le contemporain et le grand rival, malgré lui bien sûr, de J.-P. Manchette, je parle de Sébastien Japrisot, pratique la fiction criminelle, mais il n'écrit pas de polar. Piège pour Cendrillon, Compartiment tueurs ou La Dame dans l'auto avec des lunettes et un fusil, qui sont à mon sens parmi les meilleurs romans policiers jamais écrits, sont des ouvrages extrêmement complexes, extrêmement machinés au niveau du récit, et graves, et menaçants, et proches de la fable cruelle par son mode de narration; mais ils se déroulent dans un continuum beaucoup plus classique et plus conceptuel. Chez Japrisot, l'intrigue, les personnages et le ton comptent infiniment plus que l'époque, la critique sociale, la dimension politique et le style.

$9 \quad$ Jan Baetens : Si le polar est ainsi lié à la sociologie et à la culture d'une certaine époque, quelles sont alors les circonstances sociales et culturelles qui permettent de comprendre l'émergence du récit policier "belge»?

10 Luc Dellisse : Retrouver son premier livre, trente ans après sa sortie, même et surtout si on le retravaille fortement, c'est découvrir le sens caché qu'on y avait introduit sans le chercher vraiment et qui soudain vous saute aux yeux. Je voulais mettre en lumière l'œuvre et la trajectoire d'une douzaine d'auteurs de grand talent, dans le contexte de l'Occupation, et cela, bien sûr, s'y trouve. Mais je découvre à présent une logique d'ensemble, une sorte de sociologie invisible qui décrit la Belgique littéraire de cette époque comme une espèce de monde perdu. Au-delà de l'examen des œuvres, Le Policier fantôme, dans sa réédition de 2017, révèle trois aspects nouveaux.

11 Le premier, c'est l'effet d'urgence, qui tient au fait que le livre de 1984 a été mené à bien en trois mois. Cela peut paraitre anecdotique, mais cela constitue un mode d'approche 
particulier. Car ce n'était pas qu'une question d'écriture, de texte qui s'allongeait sur mes cahiers, il y avait aussi ces deux ou trois cents romans qu'il fallait lire, ces milliers de pages qui défilaient sous mes yeux, pour chercher, sous une forme aussi alerte que possible, le sens. Cela a produit, me semble-t-il, cette impression de nécessité, d'urgence, de passion qui constitue en soi une grille de lecture: il faut tout comprendre, tout saisir, tout organiser, dans l'instant, dans le vif, sans quoi la vue synoptique d'un univers un peu fantasmagorique n'aura pas lieu.

12 Le deuxième, c'est que l'univers décrit faisait le grand écart avec la réalité contemporaine (la guerre). Ces dizaines d'auteurs nouveaux et pour la plupart inconnus qui se sont consacrés à la fiction policière pendant la Deuxième Guerre mondiale, portent leur regard sur un univers imaginaire assez proche en apparence du roman policier traditionnel, où il y a des domestiques, des maisons bourgeoises, des crimes en vase clos, des discussions familiales parfois sophistiquées, parfois canailles, mais toujours sur un certain ton intemporel, dans une ambiance soutenue, sans simulation $\mathrm{du}$ langage populaire dans la forme des dialogues, où il y a très peu de personnages qui ne soient pas des bourgeois ou des grands bourgeois. On a donc une espèce de fac-similé de la fiction policière traditionnelle anglaise et française, mais transposée dans un univers imaginaire, puisqu'on n'est ni en Angleterre, ni en France, puisqu'on n'est pas non plus dans le monde extérieur peuplé d'uniformes allemands et de drapeaux à croix gammée : on n'est en réalité nulle part.

13 La troisième chose qui sort des sentiers battus, c'est que la particularité principale du corpus de cette production littéraire belge des années quarante n'est pas le sujet ni le contexte (ou l'absence de contexte), c'est la langue. Je ne parle pas du fait que c'est écrit en français courant, en français d'usage, avec juste quelques connotations régionales ("tirer son plan», "savoir ouvrir une porte»), mais que c'est une langue de convention pure, principalement dans les dialogues, qui n'ont aucun souci de psychologie ou de rendu émotionnel: une langue d'information plus que de communication, une langue discursive plus que strictement narrative.

14 La fréquentation assidue de cette langue, au fil de longues lectures, laisse une impression d'artifice. Certes, aucune langue littéraire n'est naturelle; la langue de l'écriture fictionnelle est toujours peu ou prou une convention. Mais ici, on pourrait dire que c'est une convention au deuxième degré : les personnages font semblant de parler, mais en fait c'est eux qui sont parlés; les auteurs recourent au français pour écrire, mais ils se servent du français comme d'un instrument manufacturé et non comme d'une source intime. Le style en garde un caractère correct mais un peu compassé. Il n'a pas l'espèce de naturel ou de jeté, de laisser-aller volontaire, d'élégance chiffonnée d'un costume porté par Brummel, d'une écriture à la fois travaillée et spontanée. Il a quelque chose de psychorigide qui donne sa saveur d'étrangeté et d'exotisme à une production sans malice préconçue.

Jan Baetens : Pour votre enquête, vous avez fait une étude " exhaustive " du corpus : plusieurs centaines de romans, dont beaucoup de textes totalement oubliés et souvent difficilement trouvables. En même temps, vous démontrez qu'il existe clairement un modèle, et un seul, qui sous-tend l'ensemble de cette production.

Luc Dellisse : Il y a un paradigme principal au roman policier belge de langue française, durant la guerre et jusqu'aux années $46-47$, dans le prolongement de l'après-guerre: c'est le modèle narratif choisi. Il est statique. En apparence, il témoigne d'une certaine diversité, et on a l'impression que les circonstances, les auteurs, les thèmes, les décors 
varient sans fin. Mais quand on y regarde de plus près, avec un petit effort d'accommodement, on se rend compte que ce sont toujours les mêmes cases qui sont cochées. Ce sont des romans de détection, des romans d'énigme. Ils sont courts ou ultra-courts. Ils sont écrits sans grande fantaisie, sauf deux exceptions (S.A. Steeman et Jean Ray). Ils sont tous, quelle que soit l'origine régionale des auteurs, en français. Et enfin, ils ont comme principale caractéristique de ne pas prendre en compte la réalité du monde du travail et de ne faire aucune allusion à la réalité historique, cela pour des raisons évidentes - ce sont les produits de l'Occupation.

17 Jan Baetens : Cette réalité historique n'est pas uniquement belge. En fait, comme c'est souvent le cas en Belgique, c'est bien par ce qui se passe en France qu'on peut et doit comprendre l'émergence du récit d'énigme "typiquement belge».

Luc Dellisse : En 1940, l'occupation de la France a eu pour effet de créer une certaine censure, un contrôle sur le papier et donc une réduction des tirages. Il fallait négocier avec les «bureaux » pour avoir du papier. Le nombre d'exemplaires publiés n'est jamais colossal, sauf exception. On peut le vérifier à travers l'expérience d'un éditeur belge installé à Paris, ou plutôt d'un éditeur parisien de nationalité belge, Robert Denoël. Malgré son entregent, malgré ses ruses, malgré ses complaisances, malgré ses appuis (il est à la fois l'éditeur de Céline et d'Elsa Triolet) il a dû louvoyer sans arrêt pour avoir le papier nécessaire à l'édition. Même lorsqu'il veut publier celle dont il est amoureux, Dominique Rolin, il doit se battre pour faire paraitre son premier livre. La conséquence pour la Belgique de cette réduction quantitative a été de raréfier sur le territoire belge la diffusion des livres publiés en France, c'est-à-dire la quasi-totalité des livres étrangers, soit français, soit traduits en français.

Les Belges vont donc devoir relocaliser une partie de leur production éditoriale. On voit ainsi apparaître un certain nombre d'éditeurs, pas en nombre illimité mais quand même une bonne douzaine, dont Maréchal, Moorthamers, les Auteurs associés et Le Jury sont les plus connus. Ces maisons se constituent souvent sous l'impulsion d'imprimeurs qui disposent du papier et connaissent la façon de s'en servir. Elles naissent donc vraiment de la rareté et peut-être aussi d'un sentiment diffus d'autonomie, d'un retour sur la lecture, d'un goût pour la littérature d'évasion, qui incitent divers auteurs à se lancer dans le récit policier : certains qui n'avaient jamais songé à écrire et qui n'y songeront plus après la guerre - des avocats, des notaires, des représentants de commerce -, d'autres qui n'étaient pas engagés dans la fiction policière, comme Jean Ray, et qui écrivent à cette époque quelques livres quasipoliciers, d'autres encore qui par la suite abandonneront le roman policier tout en continuant à écrire, comme Thomas Owen qui se fera connaître comme auteur de littérature fantastique, ou André-Paul Duchâteau, qui deviendra scénariste de bande dessinée.

Jan Baetens : Pour vous, le modèle du roman policier belge n'est pas Simenon, mais StanislasAndré Steeman, ce qui s'explique parfaitement en raison du lien étroit entre création et circonstances historiques, cachées ou apparentes. Simenon, en effet, a déjà quitté la Belgique, il fait sa carrière en France et ailleurs. Mais quels étaient en fait le rôle et l'action de Steeman, que le public français contemporain ne connait sans doute plus que par certaines adaptations cinématographiques de ses livres, comme L'Assassin habite au 21 (Henri-Georges Clouzot, 1942) ou Quai des orfèvres (du même réalisateur, 1947)?

21 Luc Dellisse : L'expérience ne se serait peut-être pas produite, en tout cas pas de la même manière, s'il n'y avait pas eu derrière cette floraison quelque chose ou plus 
exactement quelqu'un qui fixait le modèle et en était l'aimantation principale. Si Stanislas-André Steeman n'avait pas existé. Il y a bien sûr Simenon, mais c'est un référent imaginaire et lointain. Simenon influence peu l'écriture des romans policiers belges, pour des raisons évidentes: il n'habite pas en Belgique, n'y publie pas, ne s'occupe que de lui, et le temps diégétique de ses romans se déroule en France. Le rôle de Steeman a été essentiel, à la fois comme éditeur et comme mentor. C'est lui qui a fourni l'impulsion et la maîtrise de cette singulière aventure éditoriale, en créant et en gérant une maison d'édition nommée "Le Jury " qui propose une série de fascicules, en général assez courts, plus proches d'une nouvelle que d'un roman de longueur moyenne. C'est lui qui a publié un grand nombre de gens et qui a suscité un effet d'émulation, d'attirance, de synergie, avec des résultats inespérés, en quatre ou cinq ans, mettons sept ans si on poursuit la ligne pointillée de l'Occupation jusqu'en 47 ou 48. Une centaine d'auteurs dont pratiquement aucun, à part Jean Ray et Steeman bien sûr, n'aurait existé, n'aurait publié, sans cette émulation et cette aimantation sur fond de guerre.

Jan Baetens: Des auteurs belges, des maisons d'édition belges, des lecteurs belges, d'accord. Mais est-ce que cela suffit pour qualifier cette production et cette culture, dont vous soulignez à juste titre les influences anglaises, de "belges»? Dit autrement: en quoi le roman policier traditionnel belge est-il vraiment belge?

Luc Dellisse: Tant d'auteurs en aussi peu de temps, concentrés sur un si petit territoire, et répartis en un nombre relativement petit d'éditeurs, c'est en effet singulier. Le terme de "miracle» est sans doute excessif, mais rien de semblable ne s'était produit en Belgique et ne se reproduira sans doute plus. Pour plusieurs raisons.

Stanislas-André Steeman est un cas unique dans le paysage littéraire belge, catégorie fiction policière : une sorte d'aérolithe. Si donc il y a une floraison, elle repose sur des circonstances historiques, sur le sentiment d'émulation, mais plus encore, sur le rôle personnel d'un seul homme, qui se trouve là au moment-clé. Elle doit aussi quelque chose à une certaine présence ou réalité de la nation belge, encore augmentée par le patriotisme ressenti du fait de l'Occupation, de la perte de liberté politique de cette guerre qui se prolonge et qui jusqu'en 1942 peut donner l'impression qu'elle va donner naissance à une Europe allemande fortement intégrée.

Cette dynamique est soutenue par le fait que la langue véhiculaire de la littérature à cette époque est le français, qui n'est plus la seule langue officielle, mais reste la langue d'usage sur l'ensemble du pays, en tout cas à partir d'un certain niveau d'ascension sociale ou de réussite matérielle. La France est le pays de référence principal. Paris est la capitale culturelle de la Belgique. Ce n'est plus le cas aujourd'hui. Ni pour la Flandre, qui suit ou subit des influences anglo-saxonnes, outre son propre ethnocentrisme. Ni pour la Belgique francophone, qui rêve d'une spécificité culturelle. Parler d'un roman policier «belge » n'a plus guère de sens. Ainsi l'aventure du roman policier belge estelle une réalité, mais située dans un autre espace, éclairée par une étoile qui n'existe plus que par le souvenir.

Jan Baetens : Policier, et belge, d'accord. Mais pourquoi avoir choisi le terme de "fantôme»? Parce que la plupart de ces livres et de ces auteurs sont passés aux oubliettes? Parce que le récit policier belge est téléguidé par un modèle qui reste peu connu (et que l'on risque de confondre, et partant de mal comprendre, avec des paradigmes plus prestigieux comme le whodunit anglosaxon ou le polar à la française) ? Parce qu'il pointe sans le dire vers une réalité cachée? 

paraissait révolue, déjà sortie du temps, et sans rapport avec moi, n'était pas si éloignée: moins de quarante ans. Les protagonistes n'étaient pas devenus des fantômes. Steeman était mort mais son fils, sa veuve, certains de ses amis, étaient vivants et actifs et je les ai d'ailleurs rencontrés, suite à la parution de mon livre. Simenon même était toujours là ; son dernier livre datait de trois ans. Au cours de l'une ou l'autre émission ou débat auxquels j'étais convié, je pouvais croiser Thomas Owen, Paul Kinnet, André-Paul Duchâteau et je n'avais pas l'impression de frayer avec les invités d'un Bal des Têtes : ils étaient tous sympathiques, disponibles et assez fringants. Ce n'est que lors de la réédition du Policier fantôme, trente-trois ans plus tard, que j'ai compris que j'avais été en contact avec les rescapés d'un monde englouti. archives d'une époque reculée, sans ignorer pour autant que ce passé était encore tout mêlé au présent, et sans que cela m'empêche d'aller déjeuner dans un club house avec madame Steeman, ou de faire un déplacement en voiture (une grosse Volvo un peu vintage) avec Duchâteau, ou de fumer un cigare dans le bureau cossu de Thomas Owen : cette schizophrénie explique sans doute en partie l'impression de fantôme relayée par le titre.

Mais il y avait une autre raison d'utiliser le mot fantôme : c'est l'impression de n'avoir affaire à une typologie policière bien précise. Cette abondance d'œuvres, pourtant si frappante, avait tenu à un contexte culturellement favorable et s'était produite sur une très courte période. Mais cela ne déterminait pas pour autant, en aucune manière, un courant ou une école d'écriture ou d'inspiration. Entre les univers, entre les types de personnages et les genres d'intrigues, chez les principaux auteurs concernés, il n'y a presque aucune convergence. L'écriture non plus n'a pas de grand commun dénominateur. Il n'y a pas un ton roman belge de l'occupation, une marque de fabrique, comme il y a pu avoir un ton série noire.

La figure du policier, de l'enquêteur, quand j'essayais de la fixer, dans son rapport au monde, sa présence dans la société, sa méthode d'investigation, sa classe sociale, son langage, ses mœurs, se dérobait à toute forme d'archétype. Ce côté inclassable me plaisait. Je l'ai qualifié de fantôme, peut-être parce que la Belgique elle-même m'a toujours paru fantomatique: son décor fait penser à autre chose, à un perpétuel ailleurs ; son histoire ne détermine aucune appartenance réelle ; sa langue même ne lui appartient pas. Il y a une dimension édénique dans ce flottement de la réalité, propice à une vie d'écrivain.

32

\section{INDEX}

Mots-clés: Simenon, roman policier, littérature belge 


\section{AUTHOR}

\section{JAN BAETENS}

Jan Baetens est professeur d'études culturelles à l'université de Leuven, où il s'intéresse notamment aux rapports entre texte et image dans des genres hybrides tels que la bande dessinée ou le roman-photo. Quelques publications récentes sont: Adaptation et bande dessinée (Les Impressions Nouvelles, 2020) et Une fille comme toi (JBE éditions, 2020), un "remix" d'une collection de ciné-romans-photos à partir du scénario imaginaire d'un film typique de la Nouvelle Vague.

jan.baetens@kuleuven.be 